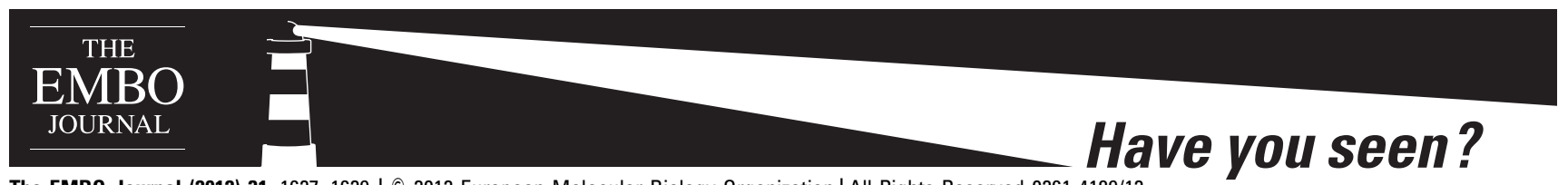

The EMBO Journal (2012) 31, 1627-1629 | (c) 2012 European Molecular Biology Organization | All Rights Reserved 0261-4189/12

www.embojournal.org

\title{
Equal opportunity for all
}

\author{
Julie Chaumeil and Jane A Skok* \\ Department of Pathology, New York University School of Medicine, New York, NY, USA \\ *Correspondence to: jane.skok@nyumc.org
}

The EMBO Journal (2012) 31, 1627-1629. doi:10.1038/emboj.2012.64; Published online 13 March 2012

By sequencing tens of millions of TCR $\alpha$ transcripts from naive mouse $\mathrm{CD8}^{+} \mathrm{T}$ cells, Genolet et al (2012) show that the $\operatorname{TCR} \alpha$ repertoire is at least as diverse as the TCR $\beta$ repertoire. This overturns a long held view in the field that recombination of the Tcra locus occurs in a co-ordinate sequential bidirectional manner that relies on proximity of $V \alpha$ and $J \alpha$ gene segments. The observation that rearrangement of all possible $\mathrm{V} \alpha-\mathrm{J} \alpha$ combinations can occur is consistent with an alternative model in which intralocus loop formation/locus contraction enables an opportunity for all $\mathrm{V} \alpha$ gene segments to recombine with $\mathrm{J} \alpha$ gene segments.

For an effective immune response, the spectrum of antigen-receptor specificity must be roughly equivalent to the spectrum of antigen diversity. To fulfill this requirement, developing $\mathrm{B}$ and $\mathrm{T}$ cells of the adaptive immune system make use of limited genomic resources to generate diversity within three Immunoglobulin (Igh, $\operatorname{Ig} \kappa$ and $\operatorname{Ig} \lambda$ ) and four T-cell receptor (Tcr $\gamma, T c r \delta, \operatorname{Tcr} \beta$ and Tcr $\alpha$ ), loci respectively. This involves co-ordinated lineage and stage-specific recombination of variable (V) diversity (D) and joining (J) genes within the different loci. To some extent, this process is like reshuffling the pieces of a puzzle, with the limitations of the design being determined by the number of available pieces that can fit together. V(D)J recombination has inbuilt restraints on joining that enables different $\mathrm{V}$, D or $\mathrm{J}$ gene segments to recombine with each other but not with members of the same family. These restrictions are determined by the type of recombination signal sequence (RSS) that is located upstream or downstream of each of the individual $\mathrm{V}, \mathrm{D}$ or $\mathrm{J}$ gene segments. The recombinase proteins, RAG 1 and 2 recognize and bind to RSSs to join together two separated gene segments through the introduction of double-strand breaks, deletion of intervening sequences and subsequent repair by the non-homologous end-joining pathway (Figure 1A). Some antigen-receptor loci such as $\operatorname{Tcr} \beta$ and Igh contain $\mathrm{D}$ as well as $\mathrm{V}$ and $\mathrm{J}$ gene segments and recombination occurs as a two-step process, which involves recombining $\mathrm{D}$ to $\mathrm{J}$ prior to $\mathrm{V}$-DJ regions. For others (Tcr $\alpha$, Igא and $\operatorname{Ig} \lambda$ ) recombination is a one step process that involves recombination of $\mathrm{V}$ to $\mathrm{J}$ gene segments. Rearrangement involving $\mathrm{V}$ and $\mathrm{J}$ gene segments alone is less restrictive and the loci can go through successive rounds of rearrangement until all available $\mathrm{V}$ or $\mathrm{J}$ gene segments are used up.
The first step of the recombination process is synapsis: RAG proteins bind to and bring together the two recombining gene segments prior to the introduction of breaks. However, since the $\mathrm{V}, \mathrm{D}$ and $\mathrm{J}$ gene segments that make up the individual germline antigen-receptor loci can occupy as much as $2.8 \mathrm{Mb}$ of DNA, individual gene segments that are potential joining partners can be separated by a large linear chromosomal expanse. Locus contraction provides a mechanism for gene segments, separated by a small or a large linear distance to recombine with an equivalent potential. This process brings widely dispersed gene segments together in nuclear space through the formation of intralocus chromatin loops (Fuxa et al, 2004; Roldan et al, 2005; Skok et al, 2007; Jhunjhunwala et al, 2009). Thus, locus contraction can impact on the ability of the immune system to mount an effective response through generating a diverse repertoire of receptors that maximizes the recombinatorial potential of each individual locus. The substructure of looping at each of the antigen-receptor loci is complex and is still being worked out (Chaumeil and Skok, 2012). Clearly, the looping conformation of the individual loci will determine what gene segments can make contacts and be joined together through the action of the RAG proteins.

The Tcra/d locus is particularly complex because in its germline form it encompasses the Tcrd locus, which recombines in $\mathrm{CD}^{-} \mathrm{CD}^{-}$double-negative (DN) T cells while Tcra rearrangement takes place at the subsequent $\mathrm{CD} 4{ }^{+} \mathrm{CD} 8^{+}$ double-positive (DP) stage of development. Productive rearrangement of $T c r \delta$ and Tcr $\gamma$ gives rise to $\gamma \delta$ T cells while a failure of their rearrangement relies on productive rearrangement of the $\operatorname{Tr} \beta$ locus to drive development forward to the DP stage where Tcr $\alpha$ rearrangement takes place. Functional rearrangement of $\operatorname{Tcr} \beta$ and Tcr $\alpha$ will generate single positive $\alpha \beta \mathrm{CD}^{+}{ }^{+}$cytotoxic and $\mathrm{CD} 4{ }^{+}$helper T cells.

Most of the Tcr $\delta$ locus (with the exception of a few $V \delta$ genes) is embedded between the $\mathrm{V} \alpha$ and $\mathrm{J} \alpha$ gene segments and thus will be deleted during Tcr $\alpha$ recombination (Figure 1B). Initiation of Tcr $\alpha$ rearrangement relies on activation of the Tcr $\alpha$ enhancer $(E \alpha)$, which in turn acts on the $T$ early activation (TEA) and $\mathrm{J}$ gene promoters to open up proximal $J \alpha$ and $V \alpha$ gene segments for primary rearrangement events (Krangel, 2003). Activation of this region of the locus depends on cohesin-mediated interaction between the TEA promoter and the E $\alpha$ (Seitan et al, 2011) (Figure 1B, black box on the right). Primary rearrangements between 
A

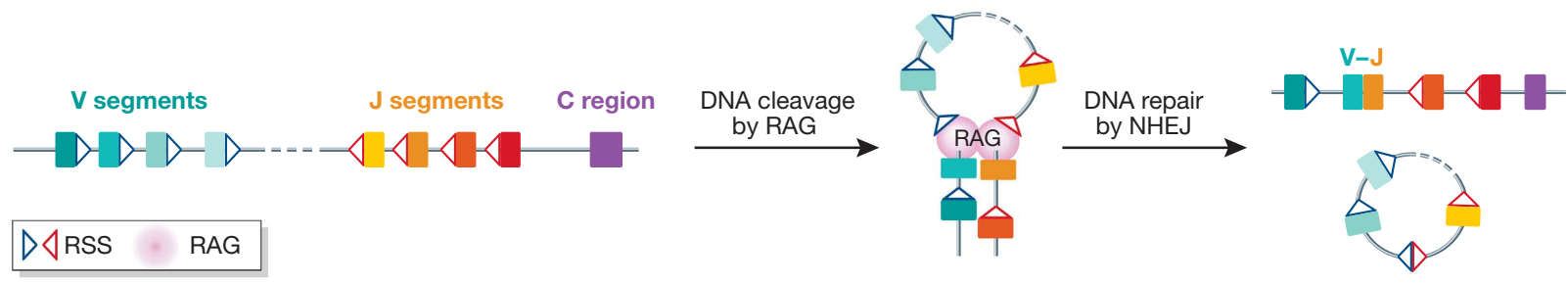

B
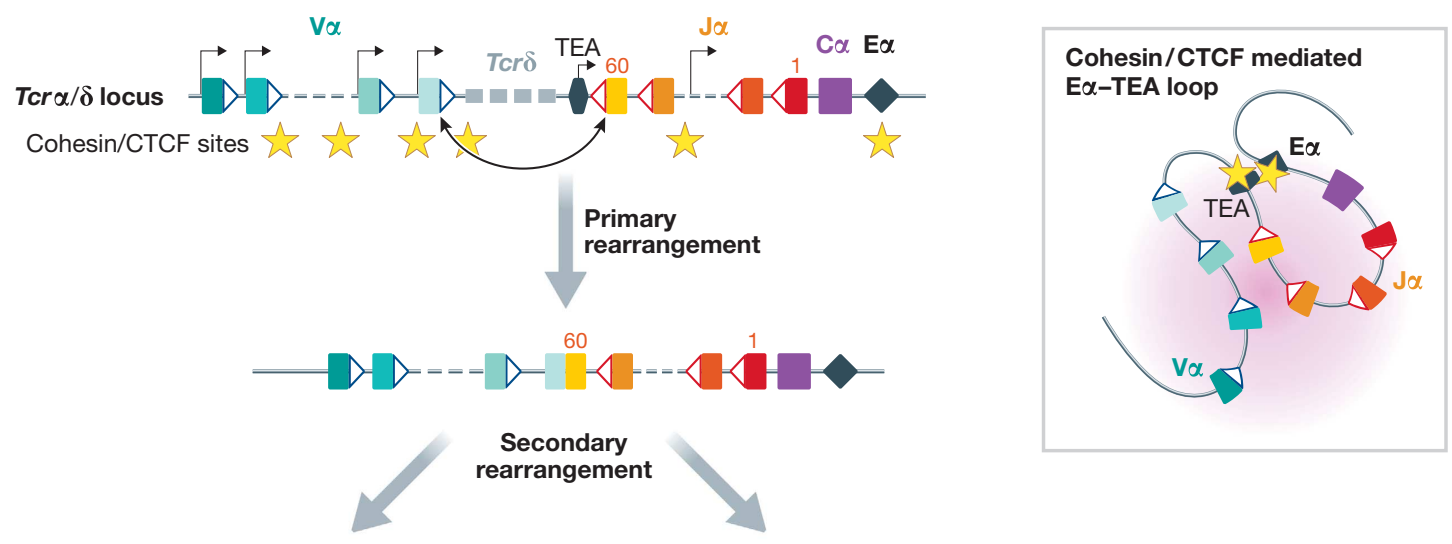

(i) Model of sequential bidirectional recombination (limited TCR $\alpha$ repertoire)

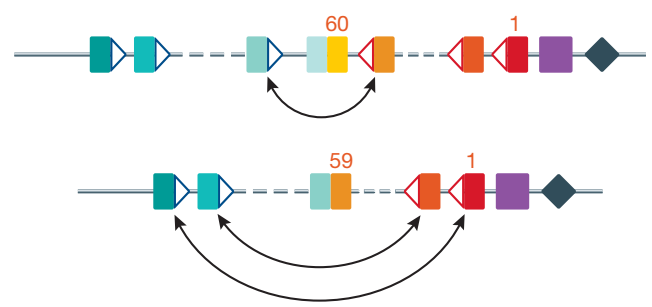

(ii) Model of locus contraction/DNA loop formation (all possible $\mathrm{V} \alpha-\mathrm{J} \alpha$ combinations)

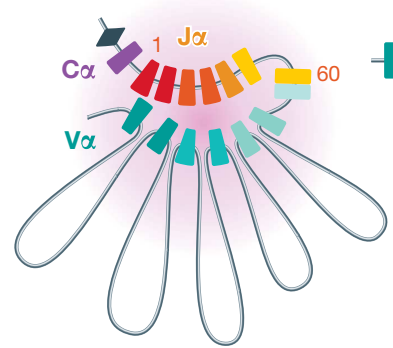

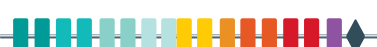

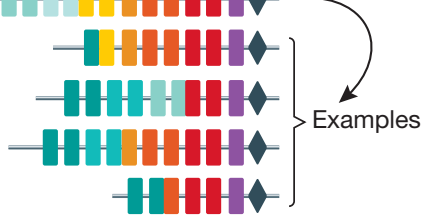

Figure 1 Recombination of the Tcra locus. (A) Outline of V(D)J recombination of antigen-receptor loci: (i) RAG1/2 recognizes, binds and cleaves DNA at specific RSSs flanking the V, D and J segments; (ii) double-stranded breaks are then repaired by the non-homologous endjoining (NHEJ) pathway. (B) In DP cells, activation of the Tcr $\alpha$ enhancer (E $\alpha$ ) and the T early activation (TEA) promoter open up proximal J $\alpha$ and $\mathrm{V} \alpha$ segments for primary rearrangement. Activation of this region depends on the formation of an E $\alpha$-TEA loop mediated by cohesin/CTCF (grey box on the right). Subsequently, Tcr $\alpha$ can go through multiple rounds of secondary rearrangement. The prevailing view is that secondary recombination occurs in a non-random bidirectional manner where $3^{\prime} \mathrm{J} \alpha$ segments join with proximal $5^{\prime} \mathrm{V} \alpha$ segments, giving rise to a limited $\mathrm{TCR} \alpha$ repertoire (i). Now sequencing of tens of millions of TCR $\alpha$ transcripts from naive mouse CD $8^{+}$T cells shows that all V $\alpha$-J $\alpha$ combinations are possible. This is consistent with an alternative model in which intralocus loop formation/locus contraction enables an equal opportunity for all $V \alpha$ segments to recombine with $\mathrm{J} \alpha$ segments (ii).

proximal $V \alpha-J \alpha$ genes will result in the deletion of the TEA and proximal $J$ gene promoters (Figure 1B). But this is not the end point of the process: in the absence of $\mathrm{D} \alpha$ gene segments, the Tcr $\alpha$ locus can go through multiple rounds of secondary rearrangement deleting previously recombined $\mathrm{V} \alpha$ and $\mathrm{J} \alpha$ gene segments.

The prevailing view is that secondary Tcr $\alpha$ recombination also occurs in a non-random manner and is governed by the position of both the $\mathrm{V} \alpha$ and $\mathrm{J} \alpha$ gene segments so that $3^{\prime} \mathrm{J} \alpha$ gene segments join with proximal $5^{\prime} \mathrm{V} \alpha$ gene segments in an ordered, coordinated bidirectional manner that is conserved in mouse and humans (Roth et al, 1991; Huang and Kanagawa, 2001; Pasqual et al, 2002; Fuschiotti et al, 2007) (Figure 1B (i)). Since rearrangement involves gene segments in close proximity, recombination does not have to rely on the formation of chromatin loops, to bring other $\mathrm{V} \alpha$ gene segments into equivalent proximity with $\mathrm{J} \alpha$ gene segments (Skok et al, 2007). Rather, ordered, position-dependent recombina- tion is compatible with a $5^{\prime}$ decontracted, $3^{\prime}$ contracted model of locus conformation that has been postulated by the Krangel laboratory (Shih and Krangel, 2010). Position biased recombination shapes a considerably more limited TCR $\alpha$ repertoire than one predicted by random recombination in which all gene segments have an equal opportunity to rearrange.

Although previous studies that support a model for a restricted TCR $\alpha$ repertoire were as far as possible designed to examine combinatorial diversity by eliminating bias, they could not cover the entire locus as comprehensively as current sequencing techniques. It is difficult to determine the actual diversity of the TCR $\alpha$ repertoire from analysing a limited fraction of $\mathrm{VJ} \alpha$ recombination events by PCR assays, which make use of a restricted range of primers. Indeed, using high throughput sequencing to examine TCR distribution in different developing and effector human T cell subsets, Wang et al (2010) arrive at an increased estimate for TCR $\alpha$ diversity. 
Now, by sequencing tens of millions of TCR $\alpha$ transcripts from naive mouse $\mathrm{CD}^{+}$T cells, Genolet et al (2012) show that the $\mathrm{TCR} \alpha$ repertoire is even greater than these estimates, and is in fact comprised of $94 \%$ of possible $\mathrm{VJ} \alpha$ combinations.

Consistent with coordinated, ordered, position biased primary rearrangement, there is infrequent representation of the most $5^{\prime} \mathrm{V} \alpha$ gene segments. These are likely deleted during secondary rearrangement and preferential rearrangement of proximal $J \alpha$ and $V \alpha$ is limited to three $V \alpha$ gene segments. The latter constitute only a minor fraction of recombinants, while the frequency of distribution of the majority of recombinants depends more on $\mathrm{V} \alpha$ gene versus $\mathrm{J} \alpha$ gene usage: $\mathrm{V} \alpha$ gene segments can recombine with nearly all $\mathrm{J} \alpha$ gene segments independent of $\mathrm{V} \alpha$ gene position, but the frequency of recombination varies in a manner that is dependent on $V \alpha$ location. Furthermore, $V J \alpha$ recombination frequencies determine the generation of diversity of $\operatorname{CDR} 3 \alpha$ (a region of the receptor that contacts antigen and is thus

\section{References}

Chaumeil J, Skok J (2012) The role of CTCF in regulating V(D)J recombination. Curr Opin Immunol 24: 1-7

Fuschiotti P, Pasqual N, Hierle V, Borel E, London J, Marche PN, Jouvin-Marche E (2007) Analysis of the TCR alpha-chain rearrangement profile in human T lymphocytes. Mol Immunol 44: 3380-3388

Fuxa M, Skok J, Souabni A, Salvagiotto G, Roldan E, Busslinger M (2004) Pax5 induces V-to-DJ rearrangements and locus contraction of the immunoglobulin heavy-chain gene. Genes Dev 18: $411-422$

Genolet R, Stevenson BJ, Farinelli L, Østerås M, Luescher IF (2012) Highly diverse $\operatorname{TCR} \alpha$ chain repertoire of pre-immune $\mathrm{CD}^{+} \mathrm{T}$ cells reveals new insights in gene recombination. EMBO J 31: 1666-1678

Huang C, Kanagawa O (2001) Ordered and coordinated rearrangement of the TCR alpha locus: role of secondary rearrangement in thymic selection. J Immunol 166: 2597-2601

Jhunjhunwala S, van Zelm MC, Peak MM, Murre C (2009) Chromatin architecture and the generation of antigen receptor diversity. Cell 138: 435-448

Krangel MS (2003) Gene segment selection in V(D)J recombination: accessibility and beyond. Nat Immunol 4: 624-630

Pasqual N, Gallagher M, Aude-Garcia C, Loiodice M, Thuderoz F, Demongeot J, Ceredig R, Marche PN, Jouvin-Marche E (2002) Quantitative and qualitative changes in V-J alpha rearrangements during mouse thymocytes differentiation: implication for a important in the immune response). These data are not consistent with a model in which secondary rearrangement occurs in a coordinated sequential position-dependent manner. Rather, the data suggest that in secondary rearrangements all $\mathrm{V} \alpha$ gene segments have equal access to the $\mathrm{J} \alpha$ region, which is compatible with a model in which contraction can occur over the entire Tcr $\alpha$ locus so that all $\mathrm{V} \alpha$ gene segments have an equivalent potential to recombine (Figure 1B (ii)). How the frequency of $\mathrm{VJ} \alpha$ recombination, which is dependent on $V \alpha$ location, is linked to a substructure of intralocus loops remains to be determined. Clearly, maximized combinatorial diversity will provide better immune coverage and influence the outcome of diseases that result from infection by foreign antigen.

\section{Conflict of interest}

The authors declare that they have no conflict of interest. limited T cell receptor alpha chain repertoire. J Exp Med 196: 1163-1173

Roldan E, Fuxa M, Chong W, Martinez D, Novatchkova M, Busslinger M, Skok JA (2005) Locus 'decontraction' and centromeric recruitment contribute to allelic exclusion of the immunoglobulin heavy-chain gene. Nat Immunol 6: 31-41

Roth ME, Holman PO, Kranz DM (1991) Nonrandom use of J alpha gene segments. Influence of $\mathrm{V}$ alpha and $\mathrm{J}$ alpha gene location. J Immunol 147: 1075-1081

Seitan VC, Hao B, Tachibana-Konwalski K, Lavagnolli T, MiraBontenbal H, Brown KE, Teng G, Carroll T, Terry A, Horan K, Marks H, Adams DJ, Schatz DG, Aragon L, Fisher AG, Krangel MS, Nasmyth K, Merkenschlager M (2011) A role for cohesin in T-cell-receptor rearrangement and thymocyte differentiation. Nature 476: 467-471

Shih HY, Krangel MS (2010) Distinct contracted conformations of the Tcra/Tcrd locus during Tcra and Tcrd recombination. J Exp Med 207: 1835-1841

Skok JA, Gisler R, Novatchkova M, Farmer D, de Laat W, Busslinger M (2007) Reversible contraction by looping of the Tcra and Tcrb loci in rearranging thymocytes. Nat Immunol 8: 378-387

Wang C, Sanders CM, Yang Q, Schroeder Jr HW, Wang E, Babrzadeh F, Gharizadeh B, Myers RM, Hudson Jr JR, Davis RW, Han J (2010) High throughput sequencing reveals a complex pattern of dynamic interrelationships among human T cell subsets. Proc Natl Acad Sci USA 107: 1518-1523 